\title{
Methods for optimal loading of the prosumer's heat source during the heating period
}

\author{
Ivan Postnikov ${ }^{1, *}$ and Andrey Penkovskii ${ }^{1}$ \\ ${ }^{1}$ Melentiev Energy Systems Institute of Siberian Branch of the Russian Academy of Sciences, \\ Laboratory of Heat Supply Systems, 664033 Irkutsk, Russia
}

\begin{abstract}
The paper the one of current problem of transition of district heating systems (DHS) to the new type of intelligent and integrated systems. This problem is related to the implementation the technology of prosumer, which allows regulating its own heat loading ensuring the most efficiency heating modes by using its own heat sources (HS) and/or heat storages. The statement of considered problem is formulated as the search of the optimal loading relation between the own distributed HS of prosumer and the district HS of DHS, based on the criterion of minimal cost on heating to prosumer for each calculated time interval during the heating period. The practical researches based on calculating experiment using the test scheme of DHS is provided. The results of calculations are presented as diagram of prosumer's and district HS loading for the considered DHS scheme, as well as the economic benefit when using distributed generation of prosumers.
\end{abstract}

\section{Introduction}

The problem of ensuring the efficient and reliable operation of district heating systems (DHS) is becoming increasingly urgent due to the development of energy technology, the growth of heat loads in cities (connecting of new consumers), as well as competition with distributed heat generation. However, despite the intense development of distributed generation, the district heating based on combined heat and power plants (CHP) is still the most energy and economic efficient technology of producing and distributing thermal energy [1-3]. At the same time, majority of DHS of cities in Europe, Russia and Asia were designed many years ago, and many technologies used in their construction no longer meet modern requirements for economy, quality and reliability of heating to consumers [4-7].

The innovative transformation of DHS, as well as other energy systems, is carried out within the framework of the concept of intelligent integrated energy (Smart Grid) [8-10]. Today, the transition of the existing DHS to the technological format of the so-called 4th generation district heating systems (4DHS) is also actively developing [10-12], suggesting of innovative technological, structural and management solutions. One of the key technology within the framework of the mentioned concepts of intelligent integrated energy systems, including 4DHS, is co-called prosumer who optimally regulate their heat

\footnotetext{
*Corresponding author: postnikov@isem.irk.ru
} 
consumption schedule by own heat source (HS) and/or heat storages, decreasing the load on district sources and network (for example for the peak of load). Most of the research on this topic is devoted to the prosumer of electric power systems [13-16], while recently the studies on the prosumer of heat energy are provided actively [17-19], including studies of the authors of this work [20-22]. In this paper, we consider the problem of optimal regulation of loading the prosumer's HS at the optimal relation with the heating from district HSs (from DHS). To solve this problem, a mathematical formalization are presented, including different models considered below.

\section{Methods}

The statement of considered problem is formulated as the search of the optimal loading relation between the own distributed HS of prosumer and the district HS of DHS, based on the criterion of minimal cost on heating to prosumer for each calculated time interval during the heating period. Mathematical formalization of this problem for each time interval (moment) $\tau$ is presented the following set of expressions:

$$
\begin{gathered}
z_{\Sigma}^{\mathrm{pro}}=\sum_{\tau \in T} \sum_{j \in J_{\mathrm{c}}^{\mathrm{pro}}}\left[c_{\tau}^{\mathrm{h}} \cdot\left(q_{j \tau}^{\mathrm{pro}}-q_{j \tau}^{\mathrm{pro}(\mathrm{hs})}\right)+z_{j \tau}^{\mathrm{pro}(\mathrm{hs})}\right] \rightarrow \min \\
\text { under next conditions and constrains: } \\
z_{j \tau}^{\mathrm{hs}}=\alpha_{j}\left(q_{j \tau}^{\mathrm{hs}}\right)^{2}+\beta_{j} q_{j \tau}^{\mathrm{hs}}+\gamma_{j}, \quad j \in J_{\mathrm{hs}} ; \\
z_{j \tau}^{\mathrm{pro(hs})}=\alpha_{j}\left(q_{j \tau}^{\mathrm{pro}(\mathrm{hs})}\right)^{2}+\beta_{j} q_{j \tau}^{\mathrm{pro}(\mathrm{hs})}+\gamma_{j}, \quad j \in J_{\mathrm{pro}(\mathrm{hs})} ; \\
z_{\tau}^{\mathrm{hnw}}=\sum_{i \in I} z_{i \tau}^{\mathrm{hnw}}=F_{1}+F_{2} \sum_{i \in I} x_{i \tau}^{2}\left|x_{i \tau}\right|_{i}, \\
f_{\mathrm{c}} \sum_{i \in I} \ell_{i}\left(a_{i}+b_{i} \ell_{i}^{0.19 u_{i}} \chi_{i}^{0.19 u_{i}} s_{i}^{-0.19 u_{i}}\right), F_{2}=c^{\mathrm{e}} /\left(367 \eta_{\mathrm{p}}\right) ; \\
q_{j \tau}^{\mathrm{c}}=q_{j}^{\mathrm{h}}\left[1-\left(1-\omega_{j}\right)\left(\tau / \tau_{\Sigma}\right)^{\sigma}\right]+q_{j}^{\mathrm{hw}}, j \in J, \tau \in T ; \\
\omega_{j}=\left(1-\varphi_{j}\right)\left[\left(t_{1}-t_{3}\right) /\left(t_{1}-t_{2}\right)\right], j \in J ; \\
v_{j}=\left(1-\varphi_{j}\right)\left[\left(t_{1}-t_{4}\right) /\left(t_{1}-t_{2}\right)\right], j \in J ; \\
c_{\tau}^{\mathrm{h}}=A C(1+R / 100 \%) ; \\
q_{j \tau}^{\mathrm{c}}=q_{j \tau}^{\mathrm{ord}}+q_{j \tau}^{\mathrm{pro}}, j \in J_{\mathrm{c}}^{\mathrm{ord}} \cap J J_{\mathrm{c}}^{\mathrm{pro}} ; \\
\left.\sigma_{j}-\omega_{j}\right) /\left(1-v_{j}\right), j \in J ;
\end{gathered}
$$




$$
\begin{gathered}
A C=\left(\sum_{j \in J_{\mathrm{hs}}} z_{j \tau}^{\mathrm{hs}}+z_{\tau}^{\mathrm{hnw}}\right) / \sum_{j \in J_{\mathrm{hs}}} q_{j \tau}^{\mathrm{hs}} \\
\mathbf{A} \mathbf{x}_{\tau}=\mathbf{g}_{\tau} \\
\overline{\mathbf{A}}^{\mathrm{T}} \mathbf{p}_{\tau}=\mathbf{h}_{\tau}-\mathbf{H}_{\tau} \\
\mathbf{S X}_{\tau} \mathbf{x}_{\tau}=\mathbf{h}_{\tau} \\
q_{j \tau}=v g_{j \tau} \Delta t
\end{gathered}
$$

Where: $c_{\tau}^{\mathrm{h}}$ - tariff for the heat supplied from the DHS, rub/GJ; $q_{j \tau}^{\mathrm{pro}(\mathrm{hs})}-$ heat selfproduction volume at the HS of the $j$-th prosumer, GJ/h; $z_{j \tau}^{\mathrm{pro}(\mathrm{hs})}-$ costs of heat selfproduction at the HS of the $j$-th prosumer, rub.; $\alpha_{j}, \mathrm{rub} /(\mathrm{GJ} / \mathrm{h})^{2}, \beta_{j}, \mathrm{rub} /(\mathrm{GJ} / \mathrm{h}), \gamma_{j}, \mathrm{rub}-$ approximation coefficients of cost characteristic of respective heat sources; $J_{\mathrm{hs}}-$ a subset of the DHS heat sources; $J_{\text {pro(hs) }}$ - a subset of prosumer HS; $F_{1}$ - semi-fixed costs, rub; $F_{2}$ - coefficient of semi-fixed costs of heat network $(\mathrm{HN})$, rub; $x_{i \tau}$ - heat carrier flow rate in the $i$-th branch of HN at time $\tau, \mathrm{t} / \mathrm{h} ; s_{i}$ - coefficient of hydraulic resistance of the $i$-th branch, $\mathrm{mh} 2 / \mathrm{t} 2 ; f_{\mathrm{c}}$ - share of semi-fixed and operational costs of HN (normally taken equal to 0.075$) ; N_{\mathrm{p}}$ - the number of pump unit operation hours, h/year; $\ell_{i}$ - length of the $i$-th network section, $\mathrm{m} ; a_{i}, b_{i}$ - approximation coefficients of costs for the $i$-th network sections with various diameters; $\chi_{i}$ - coefficient depending on the pipeline roughness of the $i$-th network section; $c^{\mathrm{e}}$ - electricity price, rub/kWh; $\eta_{\mathrm{p}}-$ pump unit efficiency, $\%$; $q_{j}^{\mathrm{h}}$ - calculated heating load, GJ/h; $q_{j}^{\mathrm{hw}}$ - calculated hot water supply load, GJ/h; $\omega_{j}, v_{j}$ and $\sigma_{j}$ - heat load curve irregularity factors; $\varphi_{j}$ - proportion of hot water supply load; $t_{1}-$ calculated indoor temperature, ${ }^{\circ} \mathrm{C} ; t_{2}, t_{3}, t_{4}$ - ambient air temperature: calculated, corresponding to the beginning of the heating season and average throughout the heating season, respectively, ${ }^{\circ} \mathrm{C} ; q_{j \tau}^{\text {ord }}$ and $q_{j \tau}^{\text {pro }}$ - heat demand of ordinary consumers and prosumers at node $j, \mathrm{GJ} / \mathrm{h} ; A C$ - specific total heat supply costs, rub/GJ; $R$ profitability, $\% ; z_{j \tau}^{\text {hs }}$ - heat production costs of the $j$-th heat source, rub; $z_{\tau}^{\text {hnw }}-$ heat transportation costs (HN operating costs), rub; $q_{j \tau}^{\mathrm{hs}}$ - volume of heat produced by the $j$-th $\mathrm{HS}, \mathrm{GJ} / \mathrm{h} ; \mathbf{A}$ - matrix of couplings of linearly independent nodes of $\mathrm{HN} ; \overline{\mathbf{A}}^{\mathrm{T}}$ - complete transposed branch and node incidence matrix; $\mathbf{x}_{\tau}$ - vector of heat carrier flow rates in the branches of $\mathrm{HN}$ at time $\tau, \mathrm{t} / \mathrm{h} ; \mathbf{g}_{\tau}$ - vector of flow rates at network nodes at time $\tau, \mathrm{t} / \mathrm{h}$; $\mathbf{p}_{\tau}$ - vector of nodal pressure in $\mathrm{HN}$ at time $\tau, \mathrm{mm} \mathrm{wc} ; \mathbf{h}_{\tau}$ - vector of head losses in the network branches at time $\tau, \mathrm{mm} \mathrm{wc} ; \mathbf{H}_{\tau}$ - vector of acting heads at HS at time $\tau, \mathrm{mm} \mathrm{wc}$; $\mathbf{S}, \mathbf{X}_{\tau}$ - diagonal matrices of coefficients of hydraulic resistance in the branches, $\mathrm{m} /(\mathrm{h} 2 \mathrm{t} 2)$, 
and absolute values of flow rates in them, t/h, at time $\tau ; v-$ heating capacity of the heat carrier, $\mathrm{GJ} / \mathrm{kg}^{\circ} \mathrm{C} ; g_{j \tau}$ - mass flow rates of a heat carrier at the $j$-th node, $\mathrm{t} / \mathrm{h} ; \Delta t$ - network water temperature difference in the supply and return pipelines, ${ }^{\circ} \mathrm{C}$.

Empirical dependencies (2) and (3) are needed to calculate the operational costs of district HS and distributed HS of prosumers at each time point [23, 24]. Formulas (4)-(5) are needed to calculate the operational costs of HS used to determine the heat tariff in DHS [25]. Equations (6)-(9) determines the schedule of heat consumption during the heating season [25]. Equations (11) and (12) determines heat tariff for each calculated time point. Model (13)-(15) and dependence (16) are needed to calculate the thermal and hydraulic conditions in HN [26]. Search for optimal solutions to the formulated problem is based on the application of the methods of univariate relaxation (the method of coordinate descent) and iterative approximation. Fig. 1 presents the interrelation stage scheme for solving the problem of optimal loading prosumer's HS is presented in the aggregate form.

\section{Case study}

The scheme of DHS under consideration is presented in Fig. 2 and consists of one district HS1 with an output of $232 \mathrm{GJ} / \mathrm{h}, 12$ consumers and a circuit $\mathrm{HN}$ consisting of 17 sections (branches). The summary load of consumers is $218 \mathrm{GJ} / \mathrm{h}$ and total length of the $\mathrm{HN}$ is 1150 $\mathrm{m}$. It is assumed that consumer 10 is a prosumer (P10) with the load $34 \mathrm{GJ} / \mathrm{h}$ and has its own HS with capacity $16 \mathrm{GJ} / \mathrm{h}$ and running on fossil fuel. We specify the quadratic cost function (3) for HS P10 with the following values of the approximation coefficients: $\alpha_{j}=$ $0.51 \mathrm{rub} / \mathrm{GJ}^{2} ; \beta_{j}=820 \mathrm{rub} / \mathrm{GJ} ; \gamma_{j}=3864 \mathrm{rub}$. The production costs of HS1 (2) are also described by a quadratic function with the following values of the approximation coefficients: $\alpha_{j}=0.47 \mathrm{rub} / \mathrm{GJ}^{2} ; \beta_{j}=750 \mathrm{rub} / \mathrm{GJ} ; \gamma_{j}=24047 \mathrm{rub}$. The duration of the calculated heating period is assumed to be $5000 \mathrm{~h}$.

Fig. 3 presents the results of multivariate calculations for each time interval $(1 \mathrm{~h})$ during the heating period, integrated into annual schedules of loading sources and the cost of heating the consumer P10.

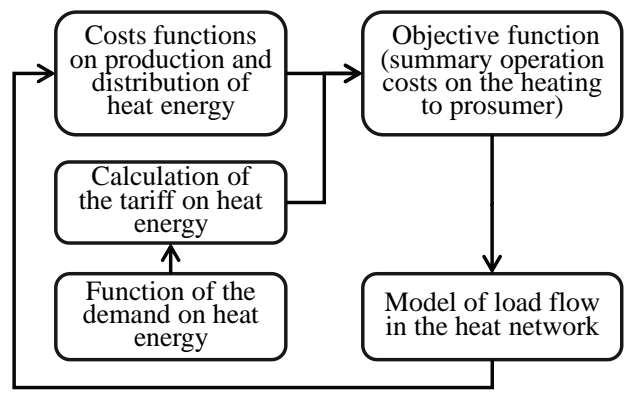

Fig. 1. Interrelation stage scheme for solving the problem of optimal loading prosumer's HS

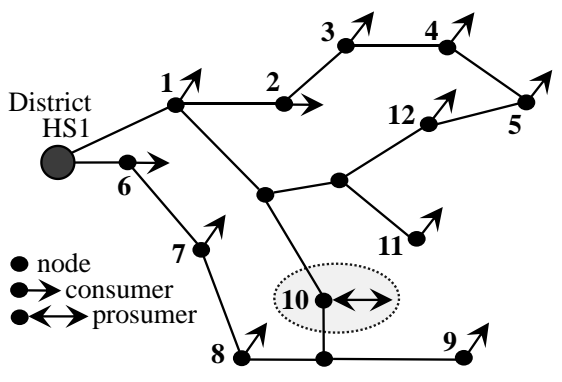

Fig. 2. Case study: considered aggregate scheme of DHS with prosumer (P10) 


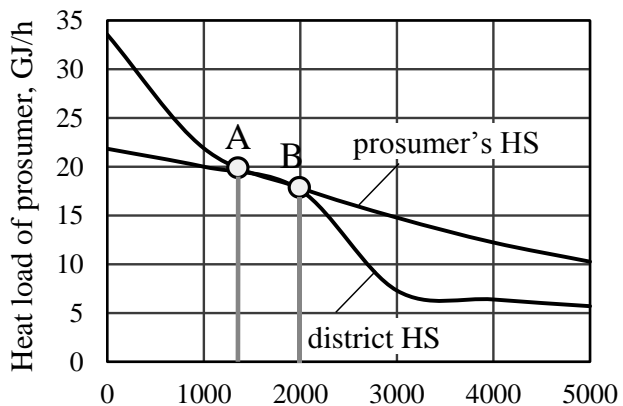

a) Time during the heating period, h/year

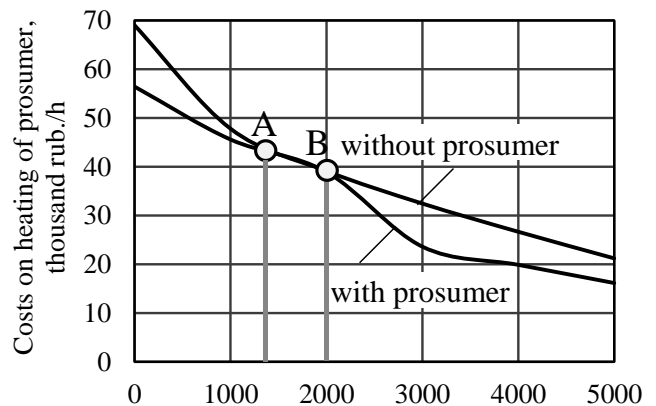

b) Time during the heating period, h/year

Fig. 3. Indices of the prosumer's HS operation in the DHS: a) optimal loading of the district HS1 and prosumer's HS for heating prosumer; $b$ ) plot of total costs of heat supply to prosumer (the area of a figure constrained by upper and bottom curves corresponds to economic benefit)

From the graph in fig. 3-a, it can be seen that the relation of loading of distributed HS P10 and district HS1 for heating prosumer P10 is distributed irregularity during the heating period. With a maximum prosumer consumption (load peak), its own source has a high load factor $(73 \%)$. Also during the load interval with a duration of 3000 to $4000 \mathrm{~h} \mathrm{HS}$ of P10 is intense used; during this period the load factor prosumer's HS is about $47 \%$. As can be seen from the graph in fig. 3-a, in the load range with a duration from 1400 to $2000 \mathrm{~h}$ (area between points A-B), the prosumer is supplied with heat energy from district HS1 completely, and its own source does not operate. This indicates that when the prosumer's heat load is in this time interval, the operating costs for its heating by its own HS and HS1 are equal or the costs of HS P10 exceeds the system costs for heating of prosumer. This is shown in the graph in Fig. 3-b, which presents the relation of the cost on heating the prosumer P10 during the calculated heating period, taking into account the participation of HS P10 in the heating, and without it. The area between the upper and lower curves represents the economic benefit achieved from the use of distributed HS of prosumer integrated into the DHS.

\section{Conclusions}

In this paper, the problem of optimal operated of the loading of own HS of prosumer in consist of DHS is formulated, which is solved in the framework of general research direction on the designing and functioning of integrated intelligent energy systems. The statement of considered problem is the search of the optimal loading relation between the own distributed HS of prosumer and the district HS of DHS, based on the criterion of minimal cost on heating to prosumer for each calculated time interval during the heating period. To solve this problem, a mathematical formalization is developed, which includes a number of mathematical models that allow taking into account different specific conditions and constrains for the systems under study. The formulated problem is solved taking into account the requirements of the thermal and hydraulic modes in HN and operating costs both sources and networks, which is a benefit of developed methods in comparison with existing approaches. Such a comprehensive consideration of all stages of the production and distribution of heat energy in DHS allows us to obtain the most reliable results corresponding to real conditions. Further research directions are mainly related to carrying out practical calculations on existing DHS schemes of cities, and solving the problem of optimization the load of distributed prosumer's HS taking into account the possibility of transferring of heat energy to a district network for heating other consumers. 
The research was performed at Melentiev Energy Systems Institute SB RAS in the framework of a scientific projects III.17.4.1 №AAAA-A17-117030310432-9 and III.17.4.3 №AAAA-A17117030310437-4 of the foundation researches program of SB RAS.

\section{References}

1. S. Werner Energy, 137(15), 617 (2017)

2. O. Pol, R.-R. Schmidt, Advanced District Heating and Cooling (DHC) Systems (Woodhead Publishing is an imprint of Elsevier, 2016)

3. U. Persson, S. Werner, Appl. Energy, 88(3), 568 (2011)

4. M. Gong, S. Werner, Energy Procedia, 116, 119 (2017)

5. B. Möller, Management of Environmental Quality: An International Journal, 19(4), 467 (2008)

6. D. Connolly, H. Lund, B. Mathiesen, et al. Energy Policy, 65, 475 (2014)

7. L. Zhang, O. Gudmundsson, H. Li, S. Svendsen, International Journal of Sustainable and Green Energy, 4(3), 102 (2015)

8. H. Lund, P. Østergaard, D. Connolly, B. Mathiesen Energy, 137, 556 (2017)

9. P. Mancarella, G. Andersson, J. Peças-Lopes, R.W. Bell K, Power Systems Computation Conference (PSCC, Genoa, Italy, 2016)

10. H. Lund, P. Østergaard, M. Chang, et al. Energy, 164, 147 (2018)

11. H. Lund, S. Werner, R. Wiltshire, et al. Energy, 68, 1 (2014)

12. H. Lund, N. Duic, P. Østergaard, B. Mathiesen, Energy, 110, 1 (2016)

13. N. Zhang, Y. Yan, W. Su, Applied Energy, 154, 471 (2015)

14. L. Perkovic, H. Mikulcic, N. Duic, Journal of Cleaner Production, 167, 1438 (2017)

15. H. Yang, T. Xiong, J. Qiu et al. Appled Energy, 167, 353 (2016)

16. R. Zafar, A. Mahmood, S. Razzaq et al. Renewable and Sustainable Energy Reviews, 82, 1675 (2018)

17. L. Brange, J. Englund, P. Lauenburg, Applied Energy, 164, 492 (2016)

18. L. Brand, A. Calvén, J. Englund et al. Applied Energy, 129, 39 (2014)

19. H. Kauko, K. Kvalsvik, D. Rohdeb, et al., Energy, 151, 261 (2018)

20. I. Postnikov, V. Stennikov, A. Penkovskii, Energy Procedia, 158, 2530 (2019)

21. I. Postnikov, V. Stennikov, A. Penkovskii, E3S Web of Conferences, 58, 1 (2018)

22. Stennikov V., Postnikov I., Penkovskii A. E3S Web of Conferences, 25, 1 (2017)

23. A. Penkovskii, V. Stennikov, O. Khamisov, E. Mednikova, I. Postnikov, Energy Procedia, 105, 3158 (2017)

24. V. Stennikov, O. Khamisov, A. Pen kovskii, Thermal Engineering, 58(12), 1043 (2011)

25. E. Sennova, V. Sidler, Mathematical modeling and optimization of developing district heating systems (Nauka, Novosibirsk, 1985)

26. A.P. Merenkov, V.Ya. Khasilev. Theory of hydraulic circuits (Nauka, Moscow, 1985) 